\title{
Study on the Curvature Reducing Method of Non-linear Regression Model
}

Jin-mei Wu, Xiao-dong Ling, Ya-wei Hou, Yu-xin Zhang, Yi Wang

China Satellite Maritime Tracking and Controlling Department, Jiangyin 214413, Jiangsu, China

\begin{abstract}
The method to reduce the non-linear strength (curvature)of non-linear regression model was studied in this paper. Firstly, the reference point of the non-linear strength was analyzed. Based on the definition of curvature cubic matrix, a computing method of curvature cubic matrix was proposed based on the Cholesky disassembling. Then the common ways to reduce the non-linear strength was also discussed. Pointed at some common non-linear models in real engineering applications, such as non-linear models used for multiple-measurement and mutual-calibration of different instruments, or non-linear models prior information, a new least square method with weight was given, which can evidently reduce the curvature of these multi-structure non-linear regression models, therefore evidently reduce the non-linear strength. Finally, the Numerical simulation results were given to validate the effectiveness and feasibility of this weighted least square method. The method to reduce the non-linear strength (curvature) of non-linear regression model was studied in this paper. Firstly, the reference point of the non-linear strength was analyzed. Based on the definition of curvature cubic matrix, a computing method of curvature cubic matrix was proposed based on the Cholesky disassembling. Then the common ways to reduce the non-linear strength was also discussed. Pointed at some common non-linear models in real engineering applications, such as non-linear models used for multiple-measurement and mutual-calibration of different instruments, or non-linear models with prior informations, a new least square method with weight was given, which can evidently reduce the curvature of these multi-structure non-linear regression models, therefore evidently reduce the non-linear strength. Finally, the Numerical simulation results ware given to validated the effectiveness and feasibility of this weighted least square method.
\end{abstract}

Keywords: Non-linear regression, curvature, cholesky disassembling, least square with weight.

\section{Introduction}

Because of the complexity of non-linear function, currently the parameter estimate of non-linear regression mainly solved by linear approximating. The precision of the estimate 
result rests with the approximate level with linear model . That is the. Beale put forward the measurement of non-linear strength firstly in [1]. Bates and Watts defined inherent curvature and parameter effect curvature in $1980^{[2]}$. Their definition applied successfully. Document [3] put forward the concept of curvature cubic matrix. We are investigating how to reduce curvature of non-linear model.

\section{Definition}

Non-linear regression usually can be expressed as $Y=f(X, \beta)+\varepsilon$, in which measurement

data

$Y=\left(y_{1}, y_{2}, \mathrm{~L}, y_{n}\right)^{\tau}$

,unknown

parameter $\beta=\left(\beta_{1}, \mathrm{~L}, \beta_{p}\right)^{\tau}, f(X, \beta)$ is the

non-linear function with design matrix $X$ and unknown parameter $\beta$. Measurement error $\varepsilon=\left(\varepsilon_{1}, \varepsilon_{2}, \mathrm{~L}, \varepsilon_{n}\right)^{\tau} \quad$ obeys $\varepsilon \sim\left(0, \sigma^{2} I_{n \times n}\right)$. The definition is as follows: The inherent curvature and parameter effect curvature cubic matrixes of non-linear model $Y=f(X, \beta)+\varepsilon$ are

$$
\begin{aligned}
& I_{(n-p) \times p \times p}=\left[N_{(n-p) \times p}^{\tau}\right]\left[U_{n \times p \times p}\right], \\
& P_{p \times p \times p}=\left[Q_{p \times n}^{\tau}\right]\left[U_{n \times p \times p}\right],
\end{aligned}
$$

$Y_{s i j}=\sum_{t=1}^{n} A_{s t} X_{t i j}, Y_{\mathrm{m} \times \mathrm{p} \times \mathrm{q}}=\left[A_{m \times n}\right]\left[X_{n \times p \times q}\right]$.

The QR disassembling of Matrix $V$ is the most important step. We can get form Cholesky disassembling theory that if $G=\left(g_{i j}\right)_{p \times p}$, $G=L D L^{\tau}$, as a result that

$$
\left\{\begin{array}{c}
d_{j}=g_{j j}-\sum_{r=1}^{j-i} c_{j r}^{2} d_{r}^{-1}, \\
c_{i j}=g_{i j}-\sum_{r=1}^{j-1} c_{j r} c_{i r} d_{r}^{-1},(j=1, \mathrm{~L}, p, i=j+1, \mathrm{~L}, p), \\
l_{i j}=\frac{c_{i j}}{d_{j}} .
\end{array}\right.
$$

From this easily get $\mathrm{L}$ and $\mathrm{D}$. Because $V$ is symmetry positive definite matrix, disassembling it as Cholesky disassembling theory, $V^{\tau} V=L D L^{\tau}=(L \sqrt{D})(L \sqrt{D})^{\tau}$, given $R=(L \sqrt{D})^{\tau}, \quad Q=V\left[(L \sqrt{D})^{\tau}\right]^{-1}$, that is $\mathrm{QR}$ disassembling of $V$.Curvature cubic matrix is the standard of non-linear strength. An important direction is reducing the non-linear strength of the model or reducing the curvature. Sum up the methods of reducing the curvature. We get three methods, Parameter varying , increasing sampling, least square method with weight. Document [3] pointed out that inherent curvature is the inherent characteristic of the model. It will remain unchanged if parameter varies. Parameter effect curvature relies parameter. In practice, different parameter varying should be chose suitably according to concrete problem at different models. Because parameter varying has not simple and explicit calculation method, many learned man tried different ways to reduce curvature. Document [4] put forward the method of increasing sampling and proofed that if provided enough 
sampling data, the non-linear model can approach the linear model adequately. In practice, the prior information $\forall \hat{\beta} \in \Theta$ can be defined and then model curvature will be reduced on creasing sampling. When the non-linear model approaches linear model adequately, the least square iteration method can be used to calculate $\hat{\beta}$.
Be aimed at non-linear multi-structure, least square estimation with weight can reduce curvature and raise precision. Set up $\quad \rho_{i} \in[0,1], \sum_{i=1}^{m} \rho_{i}=1 . \quad$ Non linear multi-structure model

$$
Y(\rho)=F_{\rho}(X, \beta)+\varepsilon(\rho), \varepsilon(\rho)=\left(0, \operatorname{diag}(\underbrace{\rho_{1} \sigma_{1}^{2}, \cdots, \rho_{1} \sigma_{1}^{2}}_{n_{1}}, \underbrace{\rho_{2} \sigma_{2}^{2}, \cdots, \rho_{2} \sigma_{2}^{2}}_{n_{2}}, \cdots, \underbrace{\rho_{m} \sigma_{m}^{2}, \cdots, \rho_{m} \sigma_{m}^{2}}_{n_{m}})\right) .
$$

The discussion about the existence and superiority of the optimal weighting can be referred to document [5].

\section{Example}

Grow model is the most wide applied model in agriculture biological、 economy v chemical. The harvest of the crops $y$ increases with $x$, but suffered from environment and weather, so we built Gompertz model: $Y_{1}=\exp \left(\beta_{1}-\beta_{2} \beta_{3}^{x}\right)+\varepsilon_{1}$, in which $\beta_{i}$ are the influencing factor we should think about. Because there are function relation between harvest and density of crops, so we can build another Holliday model. $Y_{2}=\left(\beta_{1}+\beta_{2}+\beta_{3} x^{2}\right)^{-1}+\varepsilon_{2}$. Unite the two models. We can get the non-linear multi-structure model. Set up $\varepsilon_{1} \sim N\left(0,0.01^{2}\right), \varepsilon_{2} \sim N\left(0,0.01^{2}\right)$ $\beta=\left(\beta_{1}, \beta_{2}, \beta_{3}\right)^{\tau}=(8,1,2)^{\tau}, \mathrm{x}=1,2, \ldots, 100$, and might as well $\mathrm{z}=1,2, \ldots, 100$.

Simulation the measurement data $\left\{Y_{1 i}\right\}_{i=1}^{100}$ and $\left\{Y_{2 i}\right\}_{i=1}^{100}$.We simulated 50 times and then statistic the data. Lead weight into model. Set up weights $\rho_{1}=\rho, \rho_{2}=1-\rho$, iteration initial value $(8.5,1.5,1.7)$ and step length 0.1 . Iterate with $\mathrm{G}-\mathrm{N}$ formula. The computing result is as follows.

Table 1.computed results

\begin{tabular}{|c|c|c|c|c|}
\hline & $\begin{array}{c}\text { Single structure } \\
\text { model } Y_{1}\end{array}$ & $\begin{array}{c}\text { Single structure } \\
\text { model } Y_{2}\end{array}$ & $\begin{array}{c}\text { Multi structure model } \\
\text { with Traditional } \\
\text { treatment }\end{array}$ & $\begin{array}{c}\text { Multi structure model } \\
\text { with Optimal weight }\end{array}$ \\
\hline$\rho$ & 0 & 1 & 0.5 & 0.2 \\
\hline$\hat{\beta}(\rho)$ & 7.6494 & 8.0387 & 7.9964 & 7.9989 \\
& 1.2331 & 1.0298 & 0.9973 & 0.9992 \\
& 1.4045 & 1.9793 & 2.0018 & 2.0006 \\
\hline
\end{tabular}




\begin{tabular}{|l|l|l|l|l|}
\hline $\operatorname{MSE}(\hat{\beta}(\rho))$ & 0.17726 & 0.00093 & $2.3775 E-5$ & $2.4438 E-7$ \\
& & & & \\
\hline
\end{tabular}

Table 2. curvature cubic matrix

\begin{tabular}{|c|c|c|c|c|c|c|}
\hline & \multicolumn{3}{|c|}{ Single structure model $Y_{1}$} & \multicolumn{3}{|c|}{ Single structure model $Y_{2}$} \\
\hline$\rho$ & \multicolumn{3}{|c|}{0} & \multicolumn{3}{|c|}{1} \\
\hline \multirow{9}{*}{$\begin{array}{l}\text { Parameter effect curvature } \\
\qquad P_{3 \times 3 \times 3} \text { cubic matrix }\end{array}$} & 19.1346 & -3.0727 & 0.6540 & -0.0025 & -0.0000 & 0.0000 \\
\hline & -3.0727 & 10.1343 & -3.4131 & -0.0000 & -0.0025 & 1.9827 \\
\hline & 0.6540 & -3.4131 & 4.5696 & 0.0000 & 1.9827 & -319.3462 \\
\hline & -3.0727 & 10.1343 & -3.4131 & -0.0000 & -0.0025 & 0.0000 \\
\hline & 10.1343 & 22.6259 & 2.8524 & -0.0025 & -0.0179 & 0.7496 \\
\hline & -3.4131 & 2.8524 & 21.9232 & 0.0000 & 0.7496 & -95.2534 \\
\hline & 0.6540 & -3.4131 & 4.5696 & 0.0000 & 0.0000 & -0.0025 \\
\hline & -3.4131 & 2.8524 & 21.9232 & -0.0000 & -0.0020 & -0.0457 \\
\hline & 4.5696 & 21.9232 & 54.9739 & -0.0025 & -0.0457 & -0.9965 \\
\hline & \multicolumn{3}{|c|}{$\begin{array}{l}\text { Multi structure model with } \\
\text { Traditional treatment }\end{array}$} & \multicolumn{3}{|c|}{$\begin{array}{l}\text { Multi structure model with } \\
\text { Optimal weight }\end{array}$} \\
\hline$\rho$ & \multicolumn{3}{|c|}{0.5} & \multicolumn{3}{|c|}{0.2} \\
\hline \multirow{9}{*}{$\begin{array}{l}\text { Parameter effect curvature } \\
\qquad P_{3 \times 3 \times 3} \text { cubic matrix }\end{array}$} & -0.0055 & -0.0000 & 0.0000 & -0.0035 & -0.0000 & 0.0000 \\
\hline & -0.0000 & -0.0055 & 4.4324 & -0.0000 & -0.0035 & 2.8038 \\
\hline & 0.0000 & 4.4324 & -713.7058 & 0.0000 & 2.8038 & -451.5646 \\
\hline & -0.0000 & -0.0055 & -0.0000 & -0.0000 & -0.0035 & -0.0000 \\
\hline & -0.0055 & -0.0400 & 1.6757 & -0.0035 & -0.0253 & 1.0600 \\
\hline & -0.0000 & 1.6757 & -212.8816 & -0.0000 & 1.0600 & -134.6911 \\
\hline & 0.0000 & -0.0000 & -0.0055 & 0.0000 & -0.0000 & -0.0035 \\
\hline & -0.0000 & -0.0046 & -0.1021 & -0.0000 & -0.0029 & -0.0646 \\
\hline & -0.0055 & -0.1021 & -2.2277 & -0.0035 & -0.0646 & -1.4091 \\
\hline
\end{tabular}

\section{Conclusion}

The curvature of Gompertz model is larger than the one of Holliday model. The curvature of Holliday model is very small. It explains that the Holliday model is close to linear model. The estimate result also displayed that Holliday model is more accurate than Gompertz model. The curvature of multi structure is nearly zero. It shows that multi-structure model can use all information fully to get precise least-square estimate. The method with optimal weight can get litter curvature than traditional treatment. The least square with weight can get more precise result.

\section{References}

[1] E. M. L. Beale, Confidence regions in nonlinear situation, J.R. Statist .Soc. B 22, 1960, 41-88.

[2] D.M. Bates, D.G. Watts, Relative curvature measures of nonlinearity, J. R. Statist, Soc. B 42, 1980, 1-25.

[3] Wei. Bo-cheng, Non-linear regression analysis in modern times, Southeast university press, Nanjing, 1989.

[4] Wu Yi, Yi Dongyun, Reducing curvature of a class of nonlinear regression models, Science 

in china(Series E:Technological Sciences), model with weight, Statistics and
2000(2).

[5] Wu Jinmei, Ling Xiaodong, Li Yonggang. [8] Yi Wu, Zhu Jubo. Trajectory data Least square regression model with weight for processing fusion algorithm, Science in non-linear multi-structure, Statistics and China(Series E:Technological decision, 2010(24):11-14. Sciences),1998(6).

[6] Cheng Xiru, Wang Songgui, Regression [9] Zhou Haiyin, Xie Meihua, Fusion algorithm analysis at modern times, Anhui education press, 1987.

[7] Qian Jin, Wu Jinmei, Ling Xiaodong. for error satechment based on measure data of aero-craft, Journal of Ballistics ,Vol .13 No.1 March 2001.

Computing method of least square regression 